results in subsequent investigations and to quantify this further we have reviewed our experiences.

Methods Over a 2-year period to end 2010, 236 patients were identified as investigated by PET scan after an initial discussion of all lung cancer patients at multi-disciplinary meeting (MDT). Of these, $21(11 \%)$ had high uptake FDG with potential evidence of extra thoracic disease not previously identified. Mean (range) age for this group was 69 (54-82) years with13 male and eight female.

Results Of the 21 patients, 13 included high uptake in the bowel with two in each of breast, thyroid, and musculo-skeletal and one in stomach and parotid. 4 patients were then not investigated further; these included one where PET-FDG findings were ignored after further MDT discussion, one was too unwell to investigate and died shortly thereafter, and two who refused further investigation. Of the remainder, 12 had high uptake FDG in the bowel but all were found to be benign after subsequent investigation, and five confirmed malignant disease of which two had thyroid cancer, two-breast cancer, and one had a prior history of bowel cancer. Over this 2-year time period none of the initially PET negative cases subsequently developed radiological malignancy.

Conclusion For extra thoracic disease, our experience with PET-FDG potentially reports sensitivity and a negative predictive power of $100 \%$ with a specificity of $95 \%$ and a positive predictive value of $29 \%$. Although among our cases $11 \%$ had the suggestion of positive extra thoracic disease, the majority $(71 \%)$ were then found to be benign with a concentration particularly at bowel. Further work will probably be needed to guide future investigation of potentially false positive metabolic or inflammatory findings at this site, but uptake at the other sites, potentially a concurrent primary malignancy or metastases, cannot be ignored.

\section{P198 IS THERE A ROLE FOR MRI CRANIAL IMAGING IN PATIENTS WITH NON-SMALL CELL LUNG CANCER BEING CONSIDERED FOR RADICAL TREATMENT?}

doi:10.1136/thoraxjnl-2011-201054c.198

D Laws, K Adeniji, N Ranaweera. Royal Bournemouth Hospital, Dorset, UK

Introduction Prior to undergoing radical treatment for Non-Small Cell Lung cancer (NSCLC) it is important to have accurate staging and exclude distal metastases to prevent patients undergoing futile inappropriate treatment. Guidelines ${ }^{1}$ have recommended consideration of MRI or CT imaging of the brain, especially in stage III disease. However, routine use in non-symptomatic patients has not been studied, particularly in conjunction with PET-CT scanning. In addition it is recognised that MRI is more likely to detect more and smaller lesions than CT brain. Our local policy is to offer MRI cranial imaging in all patients being considered for radical treatment in conjunction with PET scanning. We present a retrospective analysis of all these cases including follow-up and survival.

Method All patients discussed at lung cancer multi-disciplinary meeting (MDT) who were considered suitable for radical treatment were identified and offered PET-CT and MRI brain. Results of the scans, treatment received and disease free survival data were collected. Results There was data available on 115 cases. Eight were found to have cerebral metastasis on MRI scanning. $6 / 8$ (75\%) had with lung lesions of more than $3 \mathrm{~cm}$ and or a nodal status more than $\mathrm{N}>1$ on initial CT staging. All eight died within 6 months of diagnosis. 2/8 failed to meet criteria with one patient's intracranial lesions probably due to co-existing breast cancer and the other refusing biopsy and therefore may not have been NSCLC.

Conclusion Eight patients would have had radical treatment inappropriately if MRI brains had not been performed. Previous studies suggest $<10 \%$ pick up of cerebral metastases in non-symptomatic patients pre-PET scanning era. Our series shows $7 \%$. We therefore suggest a cut-off from stage $1 \mathrm{~B}-11 \mathrm{~A}$ in the 7 th TNM classification as an indication to request a contrasted MRI brain as part of the staging protocol with NSCLC where radical therapy is being contemplated.
Abstract P198 Table 1 Showing stage and demographics

\begin{tabular}{ll}
\hline Parameter & Data \\
\hline Number of patients & 115 \\
Men & 66 \\
Women & 49 \\
Age (years) & \\
Mean, (range) & $70(47-106)$ \\
Histological types & \\
Adenocarcinoma & 32 \\
Squamous cell carcinoma & 25 \\
Undifferentiated/large cell & 42 \\
Unproven/not documented & 16 \\
Diameter of primary cancer (mm) Median, (IOR) & {$[68$ Patients $>3 \mathrm{~cm}]$} \\
& {$[47$ Patients $<3 \mathrm{~cm}]$} \\
CT stage & $32,(20-47)$ \\
IA & \\
$>$ IA & 19 \\
Not documented & 86 \\
CT nodal stage & 10 \\
NO & \\
$\geq$ N1 & 63 \\
NX/Not documented & 44 \\
Synchronous metastatic brain disease & 8 \\
\hline
\end{tabular}

\section{REFERENCE}

1. Thorax 2010;65(Suppl III):iii1-27.

\section{P199 A STUDY OF SYMPTOMS AND PATHWAYS TO TREATMENT IN LUNG CANCER PATIENTS}

doi:10.1136/thoraxinl-2011-201054c.199

${ }^{1} \mathrm{U}$ Dernedde, ${ }^{2} \mathrm{R}$ Banham, ${ }^{2} \mathrm{Z}$ Tasigiannopoulos, ${ }^{2} \mathrm{~T}$ Roques, ${ }^{3} \mathrm{~A}$ Wilson, ${ }^{3} \mathrm{~A}$ Clark, ${ }^{2} \mathrm{C}$ Martin. ${ }^{1} \mathrm{James}$ Paget University Hospital, Great Yarmouth, UK; ${ }^{2}$ Norfolk and Norwich University Hospital, Norwich, UK; ${ }^{3}$ University of East Anglia, Norwich, UK

Introduction and Objectives Cancer targets promote shorter times within secondary care. However, primary care delays may be longer. Our aim was to study symptoms by asking patients themselves to record their own and any perceived delays in their pathway to treatment.

Methods 259 lung cancer patients were invited, at their first oncology visit, to complete questionnaires derived from the EORTC -LC13, including urgent and systemic symptoms and to date their start to the nearest week, when they saw their GP, whether they delayed doing so and why and how often they saw their GP before being referred for CXR or to secondary care. The GP was also mailed. Results Mean within-patient delay was 81 days (median 24). Mean delay from seeing GP to MDT meeting was 115 days (median 70), mean delay from MDT to oncology treatment was 25 days (median 15). $38 \%$ of patients admitted having delayed to see their GP. Commonest reasons were worry, denial, being too busy or family reasons. $38 \%$ patients were referred for $\mathrm{CXR} /$ to secondary care on their first GP visit, $49 \%$ on 2 nd-3rdvisit, $12 \%$ four visits or more. Analysis of variance showed no relation between stage and time to oncology. Initially, cough, dyspnoea, haemoptysis, chest pain, sputum were present in $29 \%, 30 \%, 11 \%, 9 \%$ and $13 \%$ of patients respectively. However, when patients were seen at oncology, these had risen to $76 \%, 78 \%, 30 \%, 41 \%$ and $61 \%$ respectively. Staging was available in 255 patients with stage I, II, III and IV as $6 \%, 8 \%, 40 \%$ and $46 \%$ respectively. Systemic symptoms were frequent: asthenia $64 \%$, weight loss $60 \%$, anorexia $47 \%$, dry mouth $41 \%$ and altered taste $35 \%$. Anorexia, xerostomia, altered taste, pain other than chest pain, analgesic usage and dysphagia were each correlated with stage IV rather than I-III tumours (all $\mathrm{p}<0.05$ ). 


\section{Conclusions}

1) Delay within-patient was significantly longer than withinoncology $(p<0.0001)$.

2) Over $60 \%$ patients visited their GP more than once.

3) Delay and stage were unrelated in our study.

4) Chest symptoms increased considerably during the pathway to treatment.

5) A number of common symptoms were associated with advanced disease.

\section{To wheeze, or not to wheeze: is it all asthma? P200 USE OF HYPERTONIC SALINE IN BRONCHOPROVOCATION FOR THE DIAGNOSIS OF BRONCHIAL ASTHMA}

doi:10.1136/thoraxjnl-2011-201054c.200

\section{S M Abdullah Al Mamun. Shere Bangla Medical College, Barisal, Bangladesh}

Purpose To find out a simple, chief, standard \& easily available agent for bronchoprovocation \& also to determine the provocating dose of hypertonic saline, which will reduce at least $20 \%$ of $\mathrm{FEV}_{1}$ in bronchial asthma patient.

Methods A prospective case control study was carried out among 50 patients with bronchial asthma and 50 normal healthy control at Asthma centre in NIDCH, Bangladesh. Hypertonic saline of different concentration in doubling doses $(1.8 \%, 3.6 \%, 7.2 \%)$, sequentially from lower to higher concentration was inhaled to both group by nebuliser and the test was terminated when drop of at least $20 \%$ $\mathrm{FEV}_{1}$ had occurred. Patients were selected according to prefixed inclusion \& exclusion criteria. Structured questionnaire was filled-up by each patient. Written consent was taken from every patient \& control after proper explanation about the procedure \& its outcome. Results In the control group M/F ratio was 1.27:1, age ranged from 11 years to 50 years and of 5 different occupations (student, service holder, businessman, housewife and worker). In patient group M:F was 1.38:1, age range from 11 to 50 years and five different occupation was same as control. There were no statistically significant differences between the two groups regarding age, sex and occupation. In control, graded amount of hypertonic saline $(1.8 \%, 3.6 \%, 7.2 \%)$ was given by nebuliser inhalation. None of them showed fall of $\mathrm{FEV}_{1}$ significantly $(20 \%)$. But in patient group, with similar concentration of hypertonic saline inhalation, there was highly significant fall in $\mathrm{FEV}_{1}, \chi^{2}$ test showed highly significant value, $\chi^{2}=75.42$ and $p<0.001$. Conclusion Hypertonic saline induced broncho provocation is actually very safe, simple \& cheap. So the people of third world countries can afford this test in minimum cost. Provocative dose of Hypertonic saline is $7.2 \%\left(20 \%\right.$ fall of $\left.\mathrm{FEV}_{1}\right)$.

Clinical implications For the diagnosis of cough variant asthma \& also in the suspected cases of bronchial asthma, we can performed this test confidently.

\section{P201 NO ABSTRACT ASSIGNED TO THIS NUMBER}

doi:10.1136/thoraxjnl-2011-201054c.201

\section{P202 IMPACT STUDY OF 243 INDIRECT BRONCHIAL PROVOCATION TESTS WITH MANNITOL IN THE DIAGNOSIS AND MANAGEMENT OF ASTHMA}

doi:10.1136/thoraxjnl-2011-201054c.202

I A Du Rand, L O'Reilly, D Wilson. University Hospitals Birmingham, Queen Elizabeth, Birmingham, UK

Introduction and Objective Our lung investigation unit introduced indirect bronchial provocation challenge tests with mannitol to replace direct bronchial provocation tests with Methacholine.
Mannitol challenge tests have practical and safety profile advantages. The sensitivity and specificity for $\mathrm{PC}_{20}$ Methacholine are $91 \%$ $(84.2 \%-97.8 \%)$ and $90 \%(76.9 \%-100 \%)$ respectively. ${ }^{1}$ The specificity of $\mathrm{PD}_{15}$ Mannitol compares well to Methacholine at $98.4 \%$ $(96.2 \%-99.4 \%)$, but the sensitivity of $\mathrm{PD}_{15}$ Mannitol is lower at $58.8 \%(50.7 \%-62.6 \%)^{2}$ The aim of the study was to review the clinical interpretation of mannitol challenge test results in the diagnosis of asthma.

Methods Data were collected on all Mannitol challenge tests performed between July 2008 and January 2011. A retrospective analysis of case notes was performed to assess the indication for the test, the interpretation of results and any subsequent changes in management.

Results 243 tests were performed and 240 sets of data analysed, 3 sets of case notes could not be obtained. 147 (61\%) patients presented with wheeze and dyspnoea with a possible diagnosis of asthma, 48/ $134(36 \%)$ tests were positive confirming the diagnosis and $13(8.8 \%)$ patients were unable to perform the test. $89(37 \%)$ patients presented with cough, 20/86 (23.3\%) tests were positive and three patients were unable to perform the test. $68 / 69$ (99\%) of the positive mannitol tests were interpreted as confirmation of the diagnosis of asthma. The 155 negative tests were interpreted as false negative in $20(13 \%)$ patients. In 87 (56\%) cases additional tests were subsequently performed and an alternative diagnosis was made and in 48 (31\%) cases the result was interpreted as true negative. Three of these patients $(6 \%)$ represented and were subsequently diagnosed with asthma.

Conclusion Mannitol challenge tests are useful in confirming the diagnosis of asthma in patients with high pre-test probability of the disease. Physicians need to recognise the risk of false negative mannitol test results and perform additional tests when the diagnosis is uncertain and clinical suspicion remains high.

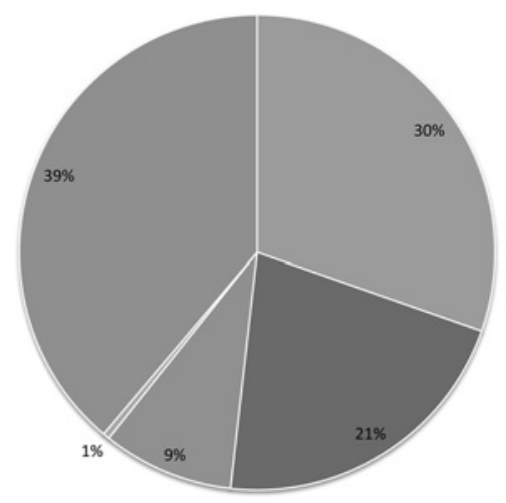

16 patients were unable to perform or complete the test (16/240) = Confirms the clinical diagnosis of asthma (68/224)

- Excludes the diagnosis of asthma (48/224) = Results interpreted as false negative and patient treated for asthma $(20 / 224)$ = Marginal positive test, asthma not diagnosed (1/224)

= Diagnosis unlikely to be asthma, an alternative diagnosis made $(87 / 224)$

Abstract P202 Figure 1 Interpretation of 240 mannitol test results.

\section{REFERENCES}

1. Hunter CJ, Brightling CE, Woltmann G, et al. A comparison of the validity of different diagnostic tests in adults with asthma. Chest 2002; 121:1051-7.

2. Sverrild A, Porsbjerg C, Thomsen SF, et al. Diagnostic properties of inhaled mannitol in the diagnosis of asthma: a population study. J Allergy Clin Immunol 2009; 124:928-32.e1.

\section{P203 CORRELATION OF NIJMEGEN SCORE AND HOSPITAL ANXIETY/DEPRESSION (HAD) SCORE IN DYSFUNCTIONAL BREATHLESSNESS}

doi:10.1136/thoraxjnl-2011-201054c.203

A Dwarakanath, V Davison, C M Taylor, A G Fennerty. Harrogate and District NHS Foundation Trust, Harrogate, UK

Introduction Dysfunctional Breathlessness has an incidence of about $10 \%$ among the general population and can often coexist with other chronic cardio respiratory illness. Patients often have a degree of anxiety or depression and may pose a diagnostic and therapeutic 